\title{
Brasil y la integración sudamericana a comienzos del Siglo 21
}

\section{Brazil and the integration of South America at the beginning of the 2lst century}

\author{
Teresinha Mendes Marra*
}

\section{Resumen}

El trabajo ofrece un análisis histórico de las relaciones internacionales sudamericanas desde el punto de vista de los alcances y límites de la integración física y política del continente a comienzos del siglo 21. Para ello se aborda la política exterior del Brasil centrándose en el gobierno del Presidente Lula, el cambio de estrategia de Estados Unidos respecto de Brasil, las relaciones con América Latina y los avances y tropiezos en la integración de América del Sur.

Palabras clave: Brasil, política exterior, integración, América del Sur, siglo 21

\section{Abstract}

The paper offers an historical analysis of South American international relations from the standpoint of the scope and limits of physical and political integration of the continent at the beginning of the 21 st century. The study of Brazilian foreign policy focuses on President Lula's

Profesora, Universidad de Goiás, Brasil (tmarra@ucg.br).

Recibido el 4 de nero de 2008; aceptado el 5 de marzo de 2008. 
Administration, the change of strategy of United Sates vis à vis Brazil, relations with Latin America and both advances and obstacles in the field of Latin American integration.

KeY wORDS: Brazil, foreign policy, integration, South America, 21st century. 
Teresinha Mendes Marra • Brasil y la integración sudamericana...

\section{INTRODUCCIÓN}

Las relaciones internacionales son por excelencia un tema que representa los intereses del Estado en el plano internacional y cuya finalidad es satisfacer las necesidades del Estado, ya para su supervivencia o ya para atender a los intereses de sus miembros (Oliveira, $2005 ; 1)$.

Sumadas a las relaciones transnacionales, el conjunto de las relaciones interestatales constituye el medio internacional, objeto de las relaciones internacionales. Desde este punto de vista, la política exterior es uno de los aspectos de las relaciones internacionales en que más se destaca el papel del Estado. La mayoría de los investigadores brasileños pone de relieve el papel preponderante del Estado en la elaboración y aplicación de la política exterior del Brasil de la cual, salvo escasas excepciones, se encuentra al margen la sociedad civil (Oliveira, 2005; 2). Sin embargo, los estudios de la política exterior brasileña centrados en el papel del Estado como actor predominante de la integración en el sistema internacional no dejan de lado la actual internacionalización de las variables y el papel de la sociedad civil, representada por asociaciones, empresas y clases, lo que imparte una dimensión transnacional a las relaciones internacionales.

El sistema internacional se compone de Estados soberanos provistos de autoridad para dirigir sus relaciones internas y de capacidad para promover sus intereses en el plano externo. Por esta razón, el Estado no reconoce ningún ente superior en el plano externo ni uno de igual jerarquía en el plano interno. En consecuencia, el Estado existe en un mundo compuesto de otros Estados que tienen características similares, territorios definidos y que constituyen unidades políticas soberanas (Oliveira, 2005; 3).

Para Russel (según Oliveira, 2005;3) la política exterior es un área particular de la acción poltica de los gobiernos que abarca tres campos analíticamente separables: político/diplomático, militar/ estratégico y económico, que se proyecta al exterior frente a una gama de actores e instituciones gubernamentales y no gubernamentales, en los planos bilateral y multilateral.

En el presente trabajo examinaremos también el concepto de Albuquerque $(2005 ; 181)$ sobre política exterior. Para este autor, la política exterior puede concebirse como la definición de los objetivos, métodos, acciones y recursos externos que deben utilizarse para lograr y mejorar condiciones que sean favorables para la seguridad nacional, incluido el empleo de recursos de poder y la amenaza del uso de la fuerza armada.

\section{LA POLÍTICA EXTERIOR DE BRASIL}

La responsabilidad de elaborar y aplicar la política exterior del país corresponde al Ministerio de Relaciones Exteriores, a partir de las orientaciones impartidas por el Presidente de la Re- 
pública. En 1998 la Constitución de Brasil reafirmó la tradición diplomática del país, cuyos preceptos básicos son los principios de soberanía nacional, cooperación y autodeterminación de los pueblos, igualdad de los Estados, defensa de la paz y solución pacífica de las controversias, rechazo del terrorismo y el racismo y respeto de los derechos humanos. Además de estos principios, la Constitución exige el compromiso de Brasil con la integración política, económica y social y cultural de los pueblos de América Latina con vistas a crear una comunidad latinoamericana de naciones.

\section{La politica exterior del Presidente Luis Inácio Lula da Silva}

Luis Inácio Lula da Silva asumió la presidencia de la República el 1 de enero de 2003 tras obtener el $61.3 \%$ de los sufragios. El nuevo presidente recibió el mandato de regir a la nación durante cuatro años. Si se piensa que hasta entonces la presidencia había sido ejercida por sociólogos, empresarios, militares y abogados, Lula representó el anti statu quo. Sin formación universitaria, de simple sindicalista se transformó en el lider popular más importante de la historia reciente del Brasil. Fue uno de los fundadores del Partido de los Trabajadores (PT) y como candidato a la presidencia fue derrotado en tres elecciones consecutivas: 1989, 1994 y 1998. Junto con Lula llegaron al gobierno otros líderes de izquierda: el ministro jefe de la Casa Civil, el ministro encargado de los derechos humanos, una ex guerrillera en el Ministerio de Minas y Energía y un ex trotskysta, en la cartera de Hacienda. La primera plana se completó con una dirigente comunitaria, ocho sindicalistas y cuatro ex exiliados. La izquierda ya no inspiraba temor puesto que el PT se había convertido en un partido de centro izquierda, sus principales líderes habían morigerado su discurso y Lula asumió el poder con un proyecto político de corte moderado (Figuereido, 2005; 30/31). En 2006 Lula fue reelegido para un nuevo mandato de cuatro años.

La política exterior aplicada por el Gobierno de Lula se remonta al segundo mandato del presidente Fernando Henrique Cardoso. Cuando este hizo uso de la palabra en la Asamblea Nacional de Francia y luego en la Organización de las Naciones Unidas, esbozó en forma bastante explícita la política exterior del país y dijo que era partidario de una política de apertura al Medio Oriente, de reconocer el Estado palestino, de oponerse al unilateralismo, de fortalecer las Naciones Unidas y de procurar oficialmente un puesto permanente para Brasil en el Consejo de Seguridad (Teixeira, según Pereira, 2005). Esta política se hizo aún más manifiesta con el presidente Lula, al punto que la prensa estadounidense llegó a hablar de un «eje del mal» formado por La Habana, Caracas y Brasilia.

Al término del primer año de su mandato, en 2003, Lula había completado un número sin precedentes de 
Teresinha Mendes Marra • Brasil y la integración sudamericana...

viajes internacionales, con 27 países visitados, mientras que en el mismo período Cardoso solo realizó 14. La política exterior brasileña contribuyó a aumentar la autoestima de los brasileños, en el sentido de que los viajes del presidente ayudaron a mejorar la imagen del país en el exterior. Lula viajó dos veces a Estados Unidos, visitó África y Europa y fue el primer presidente de Brasil en visitar el Medio Oriente. En diversos foros criticó las normas proteccionistas aplicadas por los países ricos.

En una coyuntura de proteccionismo de los países ricos, de crisis del Mercosur y de necesidad de aumentar sus exportaciones para reducir la dependencia del capital externo, Brasil amplió sus intereses comerciales a países de gran potencial de consumo, tales como Rusia, India y China. Extendió el margen de las negociaciones bilaterales y celebró acuerdos de libre comercio con China, México, India, Rusia y Chile. En 2003 estrechó relaciones con naciones del Medio Oriente, en especial con Arabia Saudita e Irán. En África, privilegió las relaciones con Egipto y Sudáfrica.

En la Organización Mundial de Comercio y para aumentar su poder de negociación con los países ricos, junto con China, India y África, Brasil lideró la creación del Grupo de los 20. Propugnó la liberación del comercio de productos agrícolas y el término de los subsidios a los productores de Estados Unidos y de la Unión Europea. Como no estuvo dispuesto a abrir sus mercados sin restricciones a los productos no agrícolas, incomodó a los representantes estadounidenses. Las negociaciones no prosperaron. Siempre en relación con la OMC, Brasil ganó 9 de los 11 casos que se ventilaron ante ese organismo multilateral. Sus principales victorias las obtuvo sobre el algodón, con Estados Unidos, y sobre el azúcar, con la Unión Europea.

Brasil participó en la Décima Conferencia de Estados Partes en el Convenio Marco de las Naciones Unidas sobre Cambio Climático (COP-10), realizada en Argentina. El informe de la conferencia dejó a Brasil en una posición incómoda respecto del Protocolo de Kyoto que fija metas de reducción de las emisiones de gases de efecto invernadero, puesto que dejó de manifiesto que Brasil había aumentado sus emisiones de dióxido de carbono. Esto se debió principalmente a la tala indiscriminada de los bosques de la Amazonía, responsable del 70\% de las emisiones de $\mathrm{CO} 2$ del país. En busca de soluciones, en 2004 el gobierno federal creó el Programa nacional de lucha contra la tala indiscriminada, en el que participan 13 ministerios y que es coordinado por la Casa Civil.

Pese a los intentos de Brasil y Estados Unidos, las negociaciones sobre la Asociación de Libre Comercio de las Américas (ALCA), bloque de libre comercio de 34 países exceptuado Cuba, no avanzaron.

Las metas de la política exterior de Brasil para 2004 fueron: consolidar el papel de Brasil como líder de América 
del Sur, abrir nuevos mercados, ocupar un lugar destacado en la lucha contra las desigualdades entre países ricos y pobres, fortalecer el Mercosur y empeñarse en lograr la representación permanente en el Consejo de Seguridad de las Naciones Unidas.

En lo que respecta al Mercosur, la política brasileña tiene tres objetivos: permitir la apertura gradual de la economía a la economía mundial, fortalecida por la ampliación del mercado subregional y luego regional gracias a las economías de escala; enfrentar los desafíos económicos y políticos de las estrategias hegemónicas de Estados Unidos en América Latina (ALCA) y ser mundialmente reconocido como potencia media, gracias a su liderazgo político en el bloque y al tamaño de un mercado del que Brasil sería el principal centro económico industrial (BernalMeza, citado en Mendes Marra, 2004; 774).

La visita realizada por el presidente Lula a la India, acompañado de 80 empresarios, se tradujo en la firma de una serie de acuerdos en los campos espacial, cultural y turístico. Al regreso de su viaje al Asia el presidente se encontró en Suiza con los presidentes de Francia y de Chile y con el Secretario General de las Naciones Unidas, oportunidad aprovechada para crear el Fondo Internacional de Lucha contra la pobreza. También en 2004, Lula visitó China acompañado de 400 empresarios. Se firmaron varios acuerdos comerciales en los campos de la siderurgia, los transportes, los agronegocios y la aviación.
Actualmente, China está contratando pilotos brasileños debido a que carece del número suficiente de profesionales altamente calificados en ese campo de actividad para hacer frente a su crecimiento acelerado.

\section{Cambio de estrategia en la política exterior estadounidense respecto de Brasil}

Si se compara con el contenido del documento básico del Gobierno de Bush sobre directrices en materia de seguridad nacional, publicado en agosto de 2002, el viaje del Presidente de Estados Unidos a América Latina en 2005 trajo consigo un cambio fundamental en la estrategia de política exterior de este país. Según se señala en ese documento, Estados Unidos «no establecerá una relación especial con las potencias regionales emergentes» (Teixeira, 2005). El gobierno estadounidense no aplicaría políticas regionales específicas y no se relacionaría con los países a través de las potencias regionales. Sin embargo, el cambio comenzó a sentirse en 2004 con la visita de Condoleezza Rice a la región. Según Teixeira (citado en Pereira, 2005) el cambio era prioritario para el propio presidente Bush, que varió de una posición de vencedor en la Guerra Fría, arrogante, de marcado unilateralismo, a una posición bastante debilitada a causa del conflicto de Irak, un fiasco de grandes proporciones, y también por el fracaso de todos los líderes que había elegido como ejemplo 
Teresinha Mendes Marra • Brasil y la integración sudamericana...

en América Latina (Teixeira, según Pereira, 2005).

¿Quiénes eran estos líderes? Bush tuvo clara preferencia por Carlos Menem en Argentina, confió en que Vicente Fox, de México, tendría gran éxito y cifró grandes esperanzas en Álvaro Uribe, de Colombia, pero el Plan Colombia fracasó. En este contexto, el gobierno estadounidense reorientó su política exterior y otorgó a Brasil la calidad de «socio regional». Los miembros del equipo de Bush, que habían servido en los gobiernos de Reagan y Bush padre, fueron antibrasileños desde la presidencia de Fernando Henrique Cardoso. Sin embargo, al perder como interlocutores a sus líderes preferidos, Bush se vio obligado a optar por Brasil como socio.

En su análisis, Teixeira, profesor de historia contemporánea de la Universidad Federal de Rio de Janeiro, señala que:

Al contrario de Argentina, la política macroeconómica de Lula es bastante responsable: su política exterior es autónoma, pero bastante pragmática. El Congreso ha acusado a Bush de haber destruido la política latinoamericana de Bill Clinton debido a que el ALCA no prosperó, no logró un área de cooperación y, por otro lado, China se convirtió en uno de los grandes socios de los países latinoamericanos (Teixeira, según Pereira, 2005).

Brasil, Venezuela y México han suscrito importantes acuerdos con China, y hay perspectivas de que se amplíen. China suscribió un pacto militar con
Cuba, firmó un tratado de comercio para vender armamento a Venezuela y desea invertir en el campo de petróleo off-shore de ese país; y Bolivia está negociando la venta de gas a China, todo ello señales evidentes de que el hemisferio ha dejado de parcela de Estados Unidos, y considera que esta geopolítica ha sido superada.

En estas circunstancias, Bush propuso al presidente Lula suscribir un acuerdo para equipararse con China y la India, que son socios de Brasil en diversos acuerdos y acciones. Sin embargo, en la reunión de la OMC realizada en Cancún, Estados Unidos prefirió aliarse con Japón y la Unión Europea contra los países emergentes. Con esta estrategia los obligaron a crear el G-20, de países contrarios a la posición de los «grandes», que luego paralizó la Ronda de Doha.

Los países de América Latina están diversificando su modelo y sus bases, incluso en lo que respecta a la compra de equipos que Estados Unidos no están dispuestos a venderles. En este mundo globalizado en que hay nuevas potencias ascendentes, América Latina se ha asociado, entre otras potencias, con China, la Federación de Rusia y la India.

Al parecer, Estados Unidos se está percatando de que, por poderosos que sean, no pueden manipular la política mundial por su cuenta. 
Brasil y las relaciones con América Latina

Actualmente, América Latina se bate en un duelo ideológico entre dos corrientes de izquierda que han asumido el poder en varios países. Según Pretkoff $(2007 ; 49)$, ex ministro y opositor del actual gobierno venezolano, Chavez es un «stalinista primitivo", que ve la solución de los problemas sociales en la implantación de un régimen socialista. El gobierno de Lula en Brasil, que ha seguido aplicando las normas de la economía de mercado y de los regímenes democráticos, pasó a ser considerado como el referente principal de una izquierda social-demócrata del continente. Para Petkoff, es un error sostener que Evo Morales o Rafael Correa se hayan inspirado en el modelo chavista.

Ver América Latina con los ojos del Departamento de Estado americano, que acostumbran ser miopes, es no entender nada de lo que ocurre en nuestro continente. En América Latina están sucediendo cosas que tienen especificidad y características propias. Es verdad que Chávez tiene presencia en Bolivia gracias a su libreto de cheques, pero Evo Morales no es producto de Chávez sino de la historia, la cultura y la política bolivianas. En el caso de Correa, de Ecuador, muchísimo menos. No es casualidad que Ecuador no forme parte de la Alternativa Bolivariana para las Américas (ALBA), bloque compuesto de Venezuela, Cuba, Bolivia y Nicaragua. (...) Chávez realmente piensa que puede liderar un bloque formado por Rusia, China, India y Brasil contra
Estados Unidos, pero ello es delirio de un megalomaníaco. No tiene nada que ver con la realidad. El discurso de Chávez es de un anti-imperialismo vacío. Sigue vendiendo a Estados Unidos un $15 \%$ del petróleo que consumen los americanos; los tanques americanos en Irak se mueven con gasolina venezolana. La oposición del Mercosur al proyecto del ALCA, liderada por Brasil, es de un antiimperialismo mucho más concreto (Petkoff,2997;49).

Brasil es el país más grande de América Latina en extensión territorial y población. Sus relaciones con sus vecinos han sido generalmente pacíficas, sin conflictos limítrofes, lo que ha contribuido a fortalecer la imagen del continente como una de las regiones más estables del mundo. Según el Stockolm International Peace Research Institute, organismo sueco especializado en el monitoreo de los gastos militares, América Latina es la región que proporcionalmente destina menos recursos a sus fuerzas armadas, cerca de $1.4 \%$ del PIB de la región (Evelin y otros, 2007; 39). Sin embargo. esta coyuntura pacífica comenzó a alterarse en 2005 , con las grandes adquisiciones de equipo militar realizadas por el gobierno de Hugo Chávez en Venezuela.

\section{Avances y tropiezos en la integración física de América del Sur}

Al contrario de Europa cuyos pueblos convivieron, pacíficamente o no, en un espacio fijado antes de que se formara la Unión Europea, América del 
Teresinha Mendes Marra • Brasil y la integración sudamericana...

Sur aún requiere integrarse físicamente. En el continente hay cuatro grandes ejes económicos y de población. El primero de ellos es el Mercosur, que une el noreste y el centro-sur de Brasil con la cuenca del Plata. El segundo es la América del Caribe, formada básicamente por Venezuela y Colombia, que mantiene relaciones comerciales principalmente con Estados Unidos. El tercer eje es el Chile meridional, que se encuentra aislado al este por la cordillera de los Andes, al norte por el desierto de Atacama y al sur por los hielos. El cuarto es la América andina, formada por los pueblos de las alturas de los Andes y por el largo litoral del Pacífico, del Perú y de Ecuador. El desafío de la integración es aumentar la vinculación entre los cuatro ejes (Ramos y otros, 2007; 48).

El proyecto de integración exige celebrar acuerdos económicos y superar conflictos políticos. A manera de ejemplo, cabe citar lo siguiente:

- Argentina, que tiene casi 40 millones de habitantes, un producto interno bruto (PIB) de 214 mil millones de dólares e inversiones brasileñas financiadas por el Banco Nacional de Desarrollo Económico y Social (BNDES) por unos 2.3 mil millones de dólares, tiene que vencer el obstáculo que representa su conflicto con Uruguay en torno a las plantas de celulosa.

- Bolivia, con una población de 9.3 millones, un PIB de 11 mil millones de dólares e inversiones del BNDES por un monto de 100 millones de dólares, está en conflicto con Brasil debido a la nacionalización del gas y del petróleo.

- Chile, que tiene 16.5 millones de habitantes, un PIB de 145 mil millones de dólares e inversiones brasileñas del BNDES por 328 millones de dólares, tiene conflictos territoriales con Argentina, Perú y Bolivia.

- Colombia, con una población de 45 millones de habitantes, un PIB de 135 mil millones de dólares e inversiones brasileñas (BNDES) cercanas a los 27 millones de dólares, tiene la presencia de militares estadounidenses en la lucha contra el narcotráfico, además de que es un país que no cuenta con las simpatías de algunos vecinos.

- Ecuador, con 13.4 millones de habitantes, un PIB de 40.8 mil millones de dólares e inversiones brasileñas del BNDES por valor de 657 millones de dólares, enfrenta la infiltración de la guerrilla colombiana en su territorio.

- Paraguay, que cuenta con 6 millones de habitantes, un PIB de 9.1 mil millones de dólares e inversiones brasileñas a través del BNDES por 77 millones de dólares tiene problemas de contrabando y narcotráfico en la frontera con Brasil y Argentina.

- Perú tiene 28.4 millones de habitantes, un PIB de 93.3 mil millones de dólares e inversiones brasileñas del BNDES por valor de 350 millones de dólares, pero mantiene una disputa territorial con Chile y debe luchar contra la infiltración de la guerrilla colombiana.

- Uruguay, con una población de 3.3 millones de habitantes, un PIB de 
19.3 mil millones de dólares e inversiones brasileñas a través del BNDES por valor de 197 millones de dólares, tiene un sistema financiero vulnerable al lavado de dinero.

- Venezuela, con una población de 27 millones de habitantes, un PIB de 181.9 mil millones de dólares e inversiones brasileñas a través del BNDES ascendentes a 515 millones de dólares, tiene conflictos territoriales con Guyana y Colombia.

- Brasil, cuya población es de 188 millones de habitantes, y que tiene un PIB de 1.1 billones de dólares y un total de 4.4 mil millones de dólares invertidos en proyectos de integración con los vecinos de América del Sur, tiene una precaria integración física en el continente y ejerce una débil vigilancia sobre la triple frontera, que es foco de contrabando, tráfico de estupefacientes y lavado de dinero (Ramos y otros, 2007; pp.48-49, basado en fuentes del Banco Mundial, Argemiro Procopio (UnB) y CIA World Factbook).

Entre los países más poblados de América del Sur, cabe destacar en orden decreciente, Brasil, Colombia, Argentina, Perú y Venezuela. Las economías más ricas de la región son, respectivamente, las de Brasil, Argentina. Venezuela, Chile y Colombia. El PIB de Chile supera el de Perú, que figura en cuarto lugar entre los países más populosos. Pese a estas desigualdades, hay un continente en construcción. Para integrar físicamente América del Sur hay muchos proyectos en marcha en materia de carreteras, líneas ferro- viarias y de energía, e hidrovías. A continuación, se señalarán los principales proyectos, seguidos de un mapa (gráfico 1) en el que pueden verse las obras o proyectos.

1. La carretera de Boa Vista (Roraima, Brasil) a Georgetown, unirá Guyana con el resto del continente. De acuerdo con los estudios financieros, la construcción de la carretera costará 200 millones de dólares.

2. La carretera/hidrovía de Manta (Ecuador) a Manaos (Amazonas, Brasil) constituirá una alternativa para el acceso al mercado asiático de los productos de la zona franca de Manaos. Su presupuesto es de 700 millones de dólares.

3. El Corredor interoceánico del Sur tiene por finalidad aumentar las corrientes comerciales entre Brasil y Perú. Su costo es de 800 millones de dólares y la obra se encuentra en marcha.

4. La carretera hacia el norte unirá regiones aisladas de Bolivia con Perú y Brasil. Su costo se estima en 270 millones de dólares.

5. El corredor bioceánico Brasil-Bolivia-Chile, constituye una alternativa para el acceso de los productos brasileños y bolivianos al Océano Pacífico. La obra se encuentra en marcha y debe estar concluida en 2009, con un costo de 900 millones de dólares.

6. El proyecto de ferrocarril de Cascavel (Paraná, Brasil) a Encarnación (Paraguay) tiene por objeto facilitar el transporte de productos tales como soja entre Brasil y Paraguay. Aún no hay estimaciones de costo. 
Teresinha Mendes Marra • Brasil y la integración sudamericana...

7. La carretera del Mercosur tiene por objeto ampliar la circulación de mercancías entre Brasil, Argentina y Uruguay. La carretera está en construcción y su costo se estima en 8 mil millones de dólares.

energía de Uruguay o la exporte a ese país. El proyecto está en estudio y su costo se prevé en 150 millones de dólares (Ramos y otros, 2007; 48, basado en informaciones del Ministerio de Rlaciones Exteriores de Brasil).

8. El tendido eléctrico Brasil-Uruguay permitirá que Brasil importe

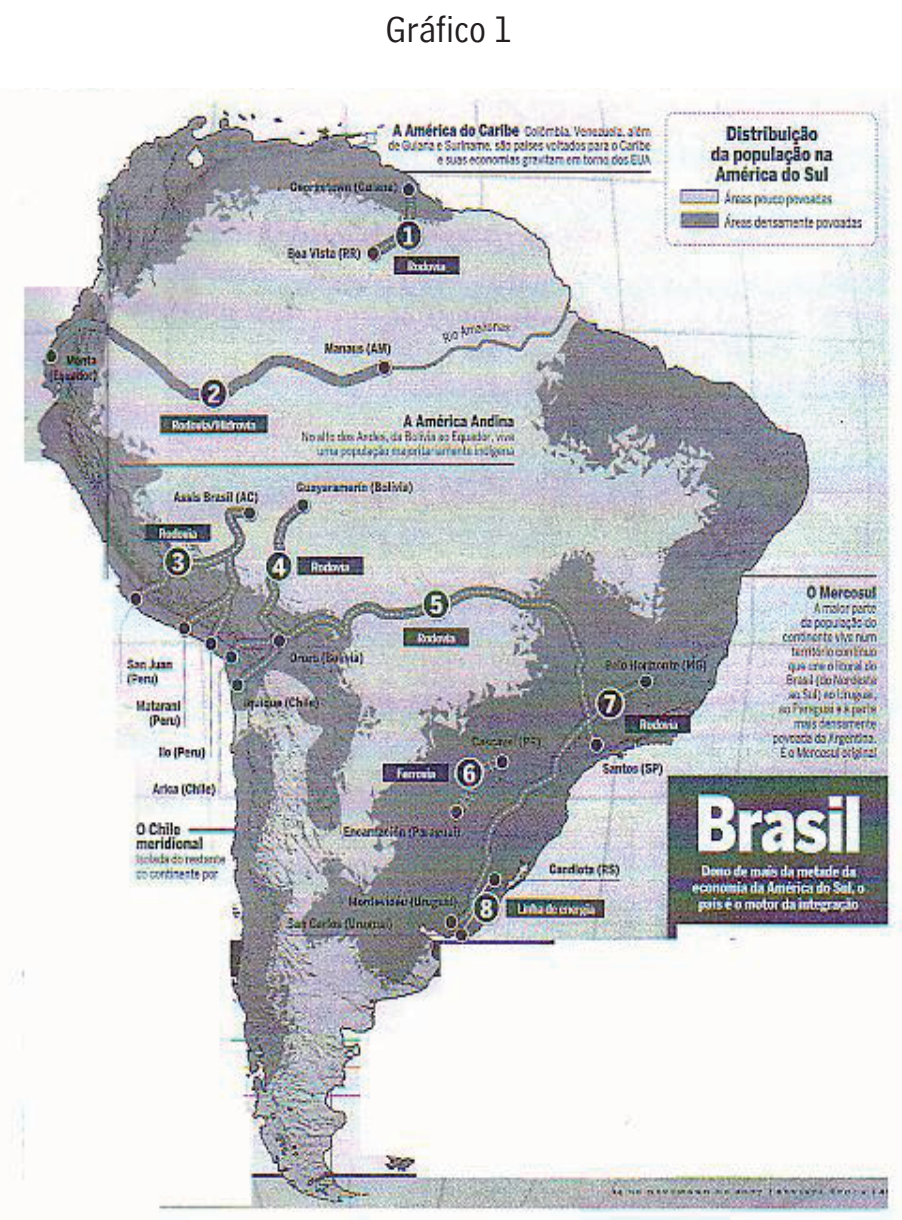

Fuente: Revista Época, 24 de 2007, p. 48. 
Como se desprende del gráfico 1, las carreteras principales son las del Mercosur y el corredor bioceánico Brasil-Bolivia-Chile. Este último, que se extiende a lo largo de 5.800 kilómetros, unirá los puertos de Santos (Brasil) y Arica (Chile). El término de los trabajos, previsto para 2009, puede situar a Bolivia en el mapa del crecimiento y de la integración de los países de Sudamérica. Para completarlo falta construir 600 kilómetros en el lado boliviano.

Además de los efectos económicos, la obra está cargada de simbolismo diplomático. Desde fines del siglo 19, cuando perdió una guerra con Chile, Bolivia reclama acceso al Océano Pacífico. Pese que no ejerce control sobre la carretera, la obra permite (permitirá) que productos bolivianos lleguen más fácilmente al mar....El Gobierno boliviano deberá obtener casi la mitad de los 900 millones de dólares que necesita para la obra mediante créditos internacionales (Ramos y otros, 2007; 51).

Favorecida por el aumento de la demanda y por el alza de los precios de los minerales y de los productos alimenticios en los principales mercados, en 2006 la economía de los países de la región creció en promedio $5.6 \%$ y atrajo grandes inversiones. Sudamèrica superó a los países árabes como destino principal de las inversiones chinas en el exterior. Con una población de 360 millones de habitantes, en 2006 el PIB sudamericano se elevó a 1.8 billones de dólares (Ramos y otros, 2007: 47).

La agenda diplomática de los países sudamericanos ha florecido al amparo de la expansión de los negocios. Se han sucedido los encuentros de presidentes para tratar cuestiones comerciales, políticas y de integración regional. A mediados de diciembre de 2007, los presidentes de Brasil, Argentina, Ecuador, Paraguay, Venezuela, Bolivia y Uruguay firmaron en Buenos Aires el acta de fundación del Banco del Sur, que pretende ser una institución de fomento como el Banco Mundial y el Banco Interamericano de Desarrollo.

En 2008 nacerá la Unión de Naciones Sudamericanas (Unasul), que según Itamaraty aspìra a ser el germen de una comunidad económica y política de países, de acuerdo con el modelo de lo que es actualmente la Unión Europea (citado por Ramos y otros, 2007; 50).

\section{Conclusiones}

En su primer año de gobierno el presidente Lula enfrentó una difícil situación. Debió esforzarse por encarar la crisis de 2002, desencadenada por las expectativas de una gestión macroeconómica de tipo heterodoxo o populista. Esto condujo a que procurara ingresar en los mercados financieros mediante la iniciativa de aumentar el superávit primario de $3.75 \%$ a $4.25 \%$ del PIB y mantener las tasas de interés en un nivel bastante elevado. Esto fue aprovechado por el sector financiero pero a expensas de un elevado costo para la población y de la reacción de los parlamentarios radicales del ala izquierda del Partido de los Trabajadores, de 
Teresinha Mendes Marra • Brasil y la integración sudamericana...

parte importante de los empresarios y del aumento de la tensión social en el campo y de vacilaciones del gobierno al momento de abordar el problema. En este período surgieron hipótesis sobre una posible giro populista, de acuerdo con el cual Lula podría profundizar su relación directa con las masas (Sallum Jr. y Kuge, 2003; 34).

En 2004 el presidente Lula se comprometió a mejorar los indicadores económicos nacionales de acuerdo con el denominado "crecimiento sustentable», tras un año de estancamiento. El gobierno apostó por una política ortodoxa, además de lo cual, se vio beneficiado por una coyuntura internacional favorable. El PIB, que en 2003 creció solo $0.5 \%$ dio un salto de casi $5 \%$ en 2004 , el desempleo se redujo y las exportaciones alcanzaron cifras sin precedentes.

En los últimos 30 meses el gobierno se ha esforzado por aumentar la importancia de Brasil en el contexto mundial. Para ello, además de lo ya señalado contribuyó a crear el Fondo para la convergencia estructural del Mercosur, con recursos por valor de 100 millones de dólares destinados a proyectos de desarrollo de las regiones menos favorecidas. A fin de integrar Brasil con los países de intereses comunes, en diciembre de 2004 el gobierno federal participó en Cuzco, Perú en la creación de la Comunidad Sudamericana de Naciones, como foro privilegiado para construir la unidad sudamericana. Profundizó su asociación estratégica con China (tercer socio comercial) para asegurar las inversiones chinas en Brasil y aumentar las exportaciones brasileñas a ese país. Con fines similares, abrió nuevas perspectivas de cooperación con los países árabes, realizando en Brasilia la Cumbre Sudamérica- países árabes. En dos años y medio el gobierno brasileño recibió la visita de 75 Jefes de Estado y abrió una oficina de Brasil en Ramalla, capital de la Autoridad Nacional Palestina, con lo cual espera contribuir a la defensa de la paz en el Medio Oriente.

Cabe destacar que uno de los principales obstáculos con que tropieza el crecimiento de la región es la falta de inversiones en infraestructura, ya que habría que invertir unos 38 mil millones de dólares. Uno de los proyectos que se considera prioritario es la unión del puerto de Manta en Ecuador con Manaos, en Brasil. El proyecto consiste en conectar las hidrovías ecuatorianas, peruanas y brasileñas, incluyendo la construcción de un conjunto de carreteras. El gobierno brasileño está estudiando la posibilidad de financiar el proyecto cuyo costo se estima en 700 millones de dólares.

La infraestructura y la burocracia no son los únicos retos que enfrenta la integración. También hay que resolver la diferencia entre las estrategias que aplican los países. Colombia, Chile y Perú quieren conservar su asociación estratégica con Estados Unidos, mientras que Argentina, Bolivia y Venezuela se pronuncian en favor del comercio interregional.

La prioridad de la Unión de Naciones Sudamericanas (Unasul), cuyo lan- 
zamiento se prevé para marzo de 2008, será la integración física y energética entre los principales polos económicos de la región.

El canciller brasileño Celso Amorim, gran defensor de la integración, está empeñado en lograr el acuerdo entre Brasil y Argentina que prevé, para el primer semestre de 2008, el uso tanto del real como del peso en las transacciones comerciales en que participan empresas brasileñas y argentinas. «Estamos liberándonos del dólar» dijo Amorim (citado en Ramos, 2007; 25). Según el Canciller, el uso de una moneda única en la Unasul, bloque que pretende integrar a los 12 paises de Sudamérica, depende del éxito de la iniciativa.

A mi juicio, pese a las críticas a la política interna, puede concluirse que la política exterior de Brasil es uno de los puntos fuertes del gobierno del presidente Lula.

\section{BibliografíA}

Albuquerque, José A. Guilhon (2005), Relaçoes Internacionais Contemporâneas. Petrópolis, Vozes.

Bernal-Meza, Raúl (2002), A política exterior do Brasil: 1990-2002, en Revista Brasileira de Política Internacional, $\mathrm{N}^{\circ} 1 \mathrm{pp}$. 35-71, Brasilia, IBRI.
Evelyn, Guilherme, Isabel Clemente y Matheus Leitao (2007), O Brasil deve ter medo dele?, en Revista Época, Nº493, São Pulo, Editora Globo.

Figueiredo, Lucas (2005), Ministerio do Silêncio, Rio de Janeiro - São Paulo, Editora Paulo, Record.

Mendes Marra, Teresinha A., Integração regional no Cone Sul; a experiência argentino-brasileira no século XX, en Revista Estudos, V. 31, Nº5, Goiânia, Editora de la Universidade Católica de Goiás.

Oliveira, Henrique Altemani (2005), Política Externa Brasileira, São Paulo, Saraiva.

Pereira, Merval (2005), Potência Regional, en Jornal O Globo, Rio de Janeiro.

Petkoff, Teodoro (2007), Lula é moderno, Chávez é primitivo, en Revista Época, No492, São Paulo, Editora Globo.

Ramos, Murilo, Ricardo Amaral y Walter Nunes (2007), O país que fala portunhol, en Revista Época, N ${ }^{\circ} 501$, São Paulo, Editora Globo.

Ramos, Murilo (2007), Adiós, dólar, en Revista Época, No 502 , São Paulo, Editora Globo.

Revista Época (2007), N501, São Paulo, Editora Globo, p. 49.

Sallum Jr. Brasilio y Eduardo Kuge (2003), Gobierno de Lula: continuidad, avance o retroceso?, en La Argentina de Kirchner y el Brasil de Lula, Carlos Chacho Alvarez (comp.), Buenos Aires, CEPES, CEDEC, Prometeo Libros.

Teixeira, Francisco Carlos (2005), Entrevista a Merval Pereira, en Jornal o Globo, Rio de Janeiro, 22 de noviembre de 2005. 